\title{
GW23-e0678 PRIMARY STUDY OF TWO STOP HYBRID IN THE TREATMENT OF MULTIVESSEL LESION IN PATIENTS WITH ACUTE CORONARY SYNDROME IN AGING
}

doi:10.1136/heartjnl-2012-302920j.55

Caiyi Lu, Changqin Gao, Yuxiao Zhang, Cangsong Xiao, Qiao Xue, Yang Wu, Wei Gao, Gang Wang, Senhua Zhou, Rui Chen, Caiyi Lu. Chinese PLA General Hospital

Objectives Evaluate the feasibility and safety of two stop hybrid in the treatment of multivessel lesion in patients with acute coronary syndrome (ACS) in aging.

Methods Two stop hybrid was adapted to treat consecutive aging patients of ACS with multivessel lesion selected by following criteria: 1. Left anterior ascending artery (LAD) was totally occluded in more than 3 months and three was at least one lesion with more than $80 \%$ stenosis in left circus flexes branch (LCX) or right coronary artery (RCA). 2. Coronary artery bypass graft was not contraindicated. 3. Informed consent was written to obtain two stop hybrid in following sequence: the patient was received micro port robot LIMA-LAD bypass at first in surgery and then transferred to cardiology in 2 weeks to complete sirolimus eluting stent implantation in LCX or RCA.

Results Five patients (male 4, 67.52 \pm 4.16 years) were enrolled in the study. Their five LADs were occluded in $15.28 \pm 4.32$ months. Three patients had 5 lesions in LCX and two had 4 lesions in RCA. Diabetes, hypertension and hyperlipidaemia were complicated in 4 , 3 and 5 cases respectively. Three were no histories of myocardial infarction and stroke. Left ventricular ejection fraction was 57.34 $\pm 6.12 \%$. Three were no death or cardiac events in 2 weeks' bridge period between surgery and cardiology. Micro port robot LIMALAD bypass were success in all five patients with patent bypass confirmed by angiography during stent implantation. Sirolimus eluting stents were implanted successfully in total 9 lesions of LCX and RCA. There were no death or cardiac events during the follow-up of $18.73 \pm 5.94$ months.

Conclusions Two stop hybrid is feasible and safe in selected aging patient of ACS with multivessel lesion. Large sample trial is needed to confirm long-term effect. 